some extent ensured, will at once command attention. Such a method has recently been worked out at the Institute of Plant Industry at Indore in Central India. The earlier results were published by Messrs. Howard and Wad in 1931 as "The Waste Products of Agriculture" which was reviewed in NATURE of November 21, 1931. In the February number of the Indian Medical Gazette of the present year, Messrs. Jackson and Wad have successfully applied the Indore method of manufacturing humus from agricultural wastes to the conversion of night soil and town refuse into a valuable compost*.

During 1932 and 1933 town wastes have been converted into humus at three centres-(1) Indore City, where the waste products of 60,000 inhabitants were dealt with; (2) the Indore Residency enclave, with a population of 4,000 ; and (3) the lines of the Malwa Bhil Corps where the numbers are about 1,000. These three centres are representative of a large municipality, a small town or military cantonment and an ordinary Indian village. The arrangements for the conversion are very simple and inexpensive. The humus factory consists of : (1) a metalled service road, $20 \mathrm{ft}$. wide; (2) a charging trench on either side, $2 \mathrm{ft}$. deep and $15 \mathrm{ft}$. wide, the floor and sides of which are preferably made smooth and impermeable so as to prevent the breeding of flies; and (3) metalled storage areas, at least $20 \mathrm{ft}$. wide, on which the ripe compost can be piled in heaps until it is sold. The manufacture of compost, which takes about a month, consists in the proper arrangement and moistening of the raw materials-town and village refuse and night soil-in the charging trench, followed by the turning of the charge three

* Institute of Plant Industry, Indore, Central India. Bulletin No. 1: The Sanitary Disposal and Agricultural Utilization of Habitation Wastes by the Indore Process. By F. K. Jackson and Y. D. Wad ; with Notes on the Sanitary Aspect by Lieut.-Col. J. R. J. Tyrrell and Lieut.-Col. M. A. Nicholson. Pp. $26+3$ plates. (Indore.) 8 annas. times at suitable intervals. An intense fermentation accompanied by a rapid rise in temperature to above $50^{\circ} \mathrm{C}$. at once sets in. The copious aeration which is ensured by the proper admixture of the materials leads to the rapid oxidation of the organic matter and to the destruction of all noxious odours, while the high temperature destroys the fly maggots and probably the ova of helminths and the spores of pathogenic bacteria as well.

The chemical composition of the final product is very satisfactory. The percentage of nitrogen on a dry basis is nearly 1 per cent while the percentages of phosphorus, potash and lime are ample. The results obtained with such crops as sugar-cane, wheat, cotton, lucerne and vegetables are such that the product finds a ready sale. The sale proceeds are considerably greater than the cost of manufacture and therefore a substantial profit is obtained, instead of the usual loss. During the last year at Indore City, for example, a net profit of Rs. 3,085 was obtained. Under the old method of disposal at this centre the net deficit was Rs. 4,535. From the point of view of sanitation and public health, two of the medical officers in Central India-Colonels Tyrrell and Nicholson-record their opinion on the process. Both consider that the method is likely to prove the most satisfactory system so far employed for the disposal of municipal wastes.

The Indore results are already being taken up at other centres in India. The process has been adopted by the Military Cantonment at Neemuch, and at Okara, a small town of 9,000 inhabitants in the Punjab. At the suggestion of Sir Malcolm Hailey, the Governor of the United Provinces, the Public Health Department has decided to experiment with the method, while the Public Works Department of New Delhi is examining the process with the view of applying it as a solution of their very serious refuse disposal problem.

\title{
Measurement of Noise
}

$\mathrm{I}^{\mathrm{N}}$ a paper read to the Institution of Electrical Engineers on March 8, Messrs. B. G. Churcher, A. J. King and H. Davies read a paper on experiments on the measurement of noise, with special reference to engineering noise problems (see also NATURE, 132, 350, Sept. 2, 1933).

The authors point out that the old conception that sounds can be classified into music and noise is untenable. For their purposes they define noise as irksome or undesired sound. For example, the sound of a radio set operated in a room to the pleasure of some of the occupants may constitute an irksome noise to others who wish to converse. They discuss the laws governing the threshold of hearing, the relation of the magnitudes of the stimuli at different frequencies which produce equal sensations of loudness and the relation between stimulus and sensation. They define the threshold as the largest sound the complete removal of which is not detected.

In determining the threshold, it is essential that there is no 'background' noise. The range of frequencies covered is $100-6,400$ cycles per second at octave intervals so that measurements were made at seven frequencies. Points determined in this way are sufficiently close to define the threshold curve.

The experiments were carried out in the laboratories of Metropolitan-Vickers Electrical Co., Ltd.
Fifty persons were experimented on and were divided into male and female groups. At 100 cycles per second the female group is $2-3$ decibels less sensitive than the male. At 800 cycles per sec. there is a tendency in both groups for sensitivity to decrease with increasing age, but the female group is now 2-3 decibels more sensitive than the male. At 6,400 cycles per sec. the average sensitivities of the two groups are approximately equal, the three oldest males having a much lower sensitivity than the rest.

The old loudness scale used by the authors and the decibel scale are logarithmic scales of physical stimulus. Doubt is thrown on the correctness of this method of measuring sound sensation. Experience has shown that the rate of increase of loudness with the decibels above the threshold is comparatively small at low intensities and much larger at high intensities. Masking and balancing methods of measuring the noise were experimentally tried and the latter was found much the more satisfactory. A pure tone was taken as the standard sound, as it is easy to specify and reproduce accurately. The procedure is to find the physical magnitude of the standard stimulus which produces a loudness sensa. tion of the same magnitude as that due to the source under observation. The judgment of loudness 
equality is much simpler than the estimate of the magnitude of loudness. It was most important that the response of the telephones used should have a linear relation with the amplitude of the disturbance especially at high values of the amplitude.

The authors have made measurements of the magnitudes of common noises on various scales. In what follows, we give them in loudness units. Calling zero the threshold of hearing, the ticking of a watch at three feet would be unity. In a quiet saloon motorcar it would be 10 . Ordinary conversation at three feet would be 20 , but if in a suburban steam train with the window open it would be 50. A loud motor horn at 100 feet was found to be 100 and two circular saws at three feet 160 .

The effect of placing the source inside a building is very pronounced. An 800 cycle tone placed in an enclosure had a loudness of 41 , whilst outside it was only $2 \cdot 4$. In making these measurements it is vitally important to take the background of noise into consideration. It is a matter of everyday experience that one sound can 'drown' another. A list of typical noise levels is given. For example, a busy main street in a certain city had a noise level of 22 . When trains were passing it rose to 53. On a weekday on the ground floor of an office in the street with the windows open, the noise level was 22 , but shutting the windows reduced it to 11 . On a Sunday morning with the windows open it was 0.6 and closed 0.2 . In a dining car in a train travelling at 60 miles per hour the level was about 50, but in a tunnel it rose to 82 .

When apparatus is installed near a main street in a busy city, we have to consider a background of between 20 and 50 . In this case a comparatively loud noise is scarcely noticed. On the other hand, when a residential hotel has to be considered, special precautions have to be taken. The screening effect produced by adjacent buildings is sometimes of assistance. At certain hours of the night the background may be so low as 1 unit and a much lower noise emission would have to be aimed at. If the problem is to be adequately dealt with in quiet residential districts, some form of enclosure must be used.

\section{Permeability Tuning in Radio-Frequency Circuits}

$\mathrm{V}$ ARIABLE condensers are now so commonly employed in radio receivers to tune circuits including a constant inductance that the use, some years ago, of variable inductances or variometers with fixed condensers is apt to be forgotten. For some purposes, however, the latter arrangement may have considerable advantages. A paper by W. J. Polydoroff ${ }^{1}$ refers to the advantages, particularly in the matter of selectivity, which result from tuning radio receiver circuits in such a manner that the ratio of the inductance to the resistance of the circuit remains constant. These desirable results may be conveniently accomplished by a new type of ferro-inductance. The coil itself is designed to have the desired performance at the highest frequency in the band to be covered. The effective inductance is then increased to tune to lower frequencies by introducing a magnetic core into the field of the coil. As the core is inserted into the coil, more lines of the magnetic field are intercepted by the core, and in effect, the average permeability of the medium surrounding the coil increases from unity, for air, to a certain maximum when the coil is entirely encased in the core: hence the term 'permeability tuning'.

The successful application of this principle to radio-frequency circuits depends upon the production of an iron-core material having an appreciable permeability at the working frequency, but free from the property of introducing undesirable resistance into the circuit. For many years, thinly laminated iron and stranded cores have been used for audio frequencies, while compressed iron dust cores have also come into use for frequencies up to about 50 kilocycles per second. Quite recently, considerable attention has been paid to the use of both iron and high permeability alloys for the construction of these dust cores, in order to obtain the necessary high permeability without the accompaniment of serious losses at radio frequencies.

In his paper, Polydoroff describes the use of pure iron reduced by hydrogen as a primary material for radio cores. While hysteresis losses are apparently vanishingly small at radio-frequencies, the eddycurrents are proportional to the square of the frequency and to the length of the circular path around each minute particle. The research described was directed at the broadcast frequency band, 550-1,500 kilocycles per second, and in this band the optimum grain size of the iron proved to be about 5 microns in diameter. This iron powder is mixed with a suitable insulating varnish and compressed in heated moulds of the desired shape, using pressures up to twenty-five tons per square inch. The resultant product has the appearance of solid iron, exhibits fair mechanical strength, and can be machined in the usual manner. The effective permeability obtained in such materials varies from about 5 to 12 according to the pressure employed in the moulding process.

The paper describes the use of this type of iron core in various types of radio receiver circuit. A good quality single layer solenoid of small dimensions is used as the inductance, and the core is made of two parts, an outer cylindrical shell and an inner plug, so as to enclose the coil in the position of maximum inductance. A semi-fixed condenser is attached to the end of each coil, and this is initially adjusted to give resonance at the highest frequency required. The cores are mounted on a common platform and inserted in their respective coils by a single tuning control. Provision is made to move each coil or each core separately in order to produce synchronisation at the middle of the range.

In receivers employing as many as six tuned circuits, no difficulty has been experienced in maintaining synchronism and constancy of the inductance to resistance ratio throughout the whole frequency band. The arrangement is equally applicable to the supersonic heterodyne and the straight radiofrequency amplifier types of receiver, and the advantages of the latter with the possibility of increased selectivity may give rise to interesting developments in the future.

1 "Ferro-Inductors and Permeability Tuning", Proc. Inst. Rad. Eng., May, 1933. 